\title{
A Note on Existence of a Bound State for a Non-Autonomous Nonlinear Scalar Field Equation
}

\author{
Liliane A. Maia ${ }^{1, *}$ and Elson L. Moura ${ }^{2}$ \\ ${ }^{1}$ Departamento de Matemática, Universidade de Brasília, 70910-900 Brasilia, Brazil \\ 2 Universidade Federal dos Vales do Jequitinhonha e Mucuri, 39803-371 Teófilo \\ Otoni-MG, Brazil
}

Received 13 March 2017; Accepted (in revised version) 9 May 2019

\begin{abstract}
The aim of this paper is to present a positive solution of a semilinear elliptic equation in $\mathbb{R}^{N}$ with non-autonomous non-linearities which are not necessarily pure-powers, nor homogeneous, and which are superlinear or asymptotically linear at infinity. The proof is variational combined with topological arguments.
\end{abstract}

Key Words: Schrödinger equation, asymptotically linear, superlinear, variational methods.

AMS Subject Classifications: 35Q55, 35B09, 35J20

\section{Introduction}

Semilinear elliptic equations in $\mathbb{R}^{N}$ arise as stationary states of Schrödinger or KleinGordon type equations, when modelling, for instance, the propagation of a light beam in Kerr and non-Kerr media, see $[2,25]$ and references therein, leading to the problem

$$
\left\{\begin{array}{l}
-\Delta u+V(x) u=f(x, u) \text { in } \mathbb{R}^{N}, \\
u \in H^{1}\left(\mathbb{R}^{N}\right) .
\end{array}\right.
$$

The search for solutions of nonlinear scalar field equations using variational methods has been intensive in the past three decades, see $[6,8,9,13,22,24]$, among many others.

The interest in this kind of problem is twofold: on one hand the large range of applications and on the other hand the mathematical challenge introduced when working in an unbounded domain like $\mathbb{R}^{N}$.

*Corresponding author. Email addresses: lilimaia@unb.br (L. A. Maia), elson.moura@ufvjm.edu.br (E. Moura) 
In this work we are mainly concerned with the following simplified version of problem $(P)$ :

$$
\left\{\begin{array}{l}
-\Delta u+u=(1+a(x)) f(u) \text { in } \mathbb{R}^{N}, \\
u \in H^{1}\left(\mathbb{R}^{N}\right),
\end{array}\right.
$$

with assumptions on $a(x)$ which imply that this problem may not have a least energy solution and it is a challenge to look for solutions in higher energy levels. Our special motivation was the notable paper of Bahri and Li [5] where they introduced a min-max procedure to prove the existence of a positive bound state solution of

$$
\left\{\begin{array}{l}
-\Delta u+u=q(x)|u|^{p-1} u \text { in } \mathbb{R}^{N}, \\
u \in H^{1}\left(\mathbb{R}^{N}\right)
\end{array}\right.
$$

where $1<p<\frac{N+2}{N-2}=2^{*}-1$, if $N \geq 3$, and $1<p<+\infty$, if $N \in\{1,2\}$ and $q \in L^{\infty}\left(\mathbb{R}^{N}\right)$ satisfying some exponential asymptotic limit, when a ground state does not exist for the problem.

Our objective is to extend [5] to non homogeneous non-linearities $f$ which are either superlinear or asymptotically linear at infinity and $a(x)$ also satisfying an exponential asymptotic limit. We use a variational approach and a topological argument introduced in [5] and updated in [12,14,19].

There is an extensive literature on this subject. We are going to highlight some articles which are more relevant with respect to our main objectives. In the autonomos cases where $V(x)=m$ and $f(x, u)=f(u)$, the pioneering work of Berestycki and Lions [9] exhibited a ground state solution for $(P)$. Using constrained minimization arguments, they showed the existence of a positive, radial solution and investigated its regularity and its exponential decay at infinity. In 1984, P. L. Lions [18] introduced breakthrough ideas of concentration-compactness that enabled numerous investigations on this subject matter.

Lehrer and Maia [17] studied problem $(P)$ with $V(x)=\lambda>0$ and $f(x, u)=a(x) f(u)$ in $\mathbb{R}^{N}$, asymptotically linear at infinity, and imposed several conditions on $a(x)$. Working in a so-called Pohozaev manifold and using a linking argument they proved existence of a bound state solution of the problem. In our work, we want to attenuate the restrictions on $a(x)$.

Clapp and Maia [12] established existence of a positive solution to the stationary nonlinear Schrödinger equation $-\Delta u+V(x) u=f(u)$ in $\mathbb{R}^{N}$ where $f$ is either superlinear or asymptotically linear at infinity using variational techniques including the case where the critical level of minimal energy is not attained. Our result is a counterpart of this together with improvent on the hypotheses.

Recently, Weth and Evequoz [14] considered the equation $(P)$ under assumptions on $a(x)$, which led them to work with the space $H^{1}\left(\mathbb{R}^{N}\right)$ under a spectral decomposition $E^{+} \oplus E^{0} \oplus E_{-}$and with $F$, the primitive of $f$, of superquadratic type at infinity. In order to 
succeed in obtaining convenient energy estimates, they had to impose other hypotheses such as : there is a constant $C>0$ such that

$$
F(x, u) \geq F_{+\infty}-C e^{-\alpha \sqrt{a_{\infty}}|x|}\left(|u|^{2}+|u|^{p}\right), \quad \alpha>0,
$$

and that $f(u)=o\left(|u|^{1+v}\right)$, as $|u| \rightarrow 0$, for some $v>0$. Thereafter, they obtained a positive solution which is not necessarily a ground state.

Inspired by the ideas in $[14,19]$, we perform some calculations of sharp energy estimates and apply a topological argument involving the barycenter function to show that there is a critical value of the functional associated with the Euler equation in $\left(P_{a}\right)$, in a suitable level of energy, giving a solution of the problem.

In contrast to the works mentioned above, we can emphasize some relevant aspects. Distinctly from the method in [5], we avoid the use of an algebraic identity (see Lemma 2.1 in [5]) by working on the constraint of the so called Nehari manifold and hence allowing for more general nonlinearities $f$ which are not homogeneous. Moreover, differently from [17], we use the Nehari manifold instead of the Pohozaev manifold enableling us to work with more general weights $a(x)$, thus a less restrictive type of problem. Finally, we were able to bypass the regularity restrictions on the function $f$ requiring only $f \in C^{1}[0,+\infty)$ by exploiting some technical calculations with our hypotheses (see Remark 1.1 and Lemma 4.1) and avoiding either the use of Lemma 2.2 in [1] or assumptions $\left(F_{2}^{\prime}\right)$ and (4) in [14]. In fact, $f$ could be less regular (see [26]), but we are going to assume more regularity in order to focus on other aspects of the problem.

To our knowledge this result is new and by examining the interactions of two copies of translations of the positive ground state solution of the limit problem

$$
\left\{\begin{array}{l}
-\Delta u+u=f(u) \quad \text { in } \mathbb{R}^{N}, \\
u \in H^{1}\left(\mathbb{R}^{N}\right),
\end{array}\right.
$$

and treating precise exponential estimates of these interactions, we are able to extend the results in [5].

We assume that $N \geq 2$ and consider $f$, not necessarily homogeneous, of this kind:

- $\left(f_{1}\right) f \in C^{1}[0,+\infty)$ and there are positive constants $C$, $p_{1}$, $p_{2}$ with $1<p_{1} \leq p_{2}<2^{*}-1$ such that $f$ and its first derivative have the growth

$$
\left|f^{(k)}(t)\right| \leq C\left(|t|^{p_{1}-k}+|t|^{p_{2}-k}\right)
$$

for $k \in\{0,1\}$ and $t>0$;

- $\left(f_{2}\right) f^{\prime}(t)>\frac{f(t)}{t}$, if $t>0$;

- $\left(f_{3}\right) \lim _{t \rightarrow+\infty} \frac{f(t)}{t} \geq l_{\infty}>1$, for some $l_{\infty} \in \mathbb{R}^{+}$; 
- $\left(f_{4}\right) f(t) t-2 F(t) \geq 0$ and $\lim _{t \rightarrow+\infty}\{f(t) t-2 F(t)\}=+\infty$, where we set $F(t):=\int_{0}^{t} f(s) d s$;

- $(U)$ The positive solution of $\left(P_{\infty}\right)$ is unique up to translations.

Moreover, we also assume the following hypotheses on the weight function:

- $\left(a_{1}\right) a \in C\left(\mathbb{R}^{N}\right) \cap L^{\infty}\left(\mathbb{R}^{N}\right) ; \inf _{x \in \mathbb{R}^{N}}(1+a(x)):=\tau>0$ and $\lim _{|x| \rightarrow+\infty} a(x)=0$;

- $\left(a_{2}\right)|a(x)| \leq C e^{-\kappa|x|}$, where $\kappa \in\left(2, p_{1}+1\right)$ for all $x \in \mathbb{R}^{N}$ and $C$ a positive constant.

Remark 1.1. Observe that the hypothesis $\left(f_{1}\right)$ implies that for every $\mu>0$ satisfying $0<1+\mu<p_{1}$ it holds that $f(u)=o\left(|u|^{1+\mu}\right)$, as $|u| \rightarrow 0$.

Remark 1.2. The assumption $(U)$ of uniqueness of the positive solution for the limit problem $\left(P_{\infty}\right)$ is known, for instance, if the nonlinearity is a pure power such as $f(s)=$ $|s|^{p}$, with $1<p<2^{*}$ by [16] or in the asymptotically linear model case $f(s)=l_{\infty} s^{3} /(1+$ $s^{2}$ ) by [23]. An assumption of type

$$
h(u):=\frac{-u+f(u)}{u f^{\prime}(u)-f(u)}
$$

is nondecreasing in $(\xi, \infty)$ where $\xi$ is the unique positive number such that $1=f(\xi) / \xi$ would suffice by [23] and [21]. In general, without some additional conditions necessary on $f$, it is not possible to guarantee the uniqueness of a positive solution of $\left(P_{\infty}\right)$.

Our main objective in this work is proving the following result.

Theorem 1.1. Assume that hypotheses $\left(a_{1}\right),\left(a_{2}\right),\left(f_{1}\right)-\left(f_{4}\right)$ and $(U)$ hold true, then problem $\left(P_{a}\right)$ has a positive classical solution $u \in H^{1}\left(\mathbb{R}^{N}\right)$.

\section{Preliminary results}

We extend $f$ as an odd function in $C^{1}(\mathbb{R})$ by defining $f(t):=-f(-t)$ for $t<0$. Note that, if $u$ is a positive solution of problem $\left(P_{a}\right)$ for the new function, $u$ is also a solution of $\left(P_{a}\right)$ for the original function $f$. Hereafter, $C>0$ will denote a positive constant and not necessarily the same one.

We will work with the canonical inner product in $H^{1}\left(\mathbb{R}^{N}\right)$ and norm defined respectively by

$$
\langle u, v\rangle:=\int_{\mathbb{R}^{N}}(\nabla u \nabla v+u v) d x, \quad\|u\|^{2}:=\int_{\mathbb{R}^{N}}\left(|\nabla u|^{2}+u^{2}\right) d x .
$$

We consider the functional associated with the problem $\left(P_{a}\right)$

$$
I(u):=\frac{1}{2}\|u\|^{2}-\int_{\mathbb{R}^{N}}(1+a(x)) F(u) d x, \quad u \in H^{1}\left(\mathbb{R}^{N}\right),
$$


so that the solutions of problem $\left(P_{a}\right)$ are the critical points of the functional $I$.

The Nehari manifold of the functional $I$ is defined by

$$
\mathcal{N}:=\left\{u \in H^{1}\left(\mathbb{R}^{N}\right) \backslash\{0\} ; J(u)=0\right\},
$$

where

$$
J(u):=I^{\prime}(u) u=\|u\|^{2}-\int_{\mathbb{R}^{N}}(1+a(x)) f(u) u d x .
$$

Furthermore, we consider the minimal level of energy of the functional $I$ given by

$$
m:=\inf _{u \in \mathcal{N}} I(u) .
$$

Analogously for $\left(P_{\infty}\right)$, we write $I_{\infty}, J_{\infty}, \mathcal{N}_{\infty}$ and $m_{\infty}$, respectively.

Our first result includes the main properties about the manifold $\mathcal{N}$.

Lemma 2.1. The following holds for the subset $\mathcal{N}$ :

a) There exists a number $\alpha>0$ such that for all $u \in \mathcal{N}$ it holds that $\|u\| \geq \alpha$;

b) $\mathcal{N}$ is a closed $C^{1}$ - submanifold of $H^{1}\left(\mathbb{R}^{N}\right)$;

c) If $u \in \mathcal{N}$, the function $t \mapsto g(t):=I(t u)$ is strictly increasing in $[0,1)$ and strictly decreasing in $(1,+\infty)$. In particular, we have $I(u)=\max _{t>0} I(t u)>0$.

Proof. a) Using $\left(f_{1}\right)$ and $J$ as in (2.3), for all $u \in \mathcal{N}$, we have

$$
\begin{aligned}
J(u) & =I^{\prime}(u) u=\|u\|^{2}-\int_{\mathbb{R}^{N}}(1+a(x)) f(u) u d x \\
& \geq\|u\|^{2}-C \int_{\mathbb{R}^{N}}\left(|u|^{p_{1}+1}+|u|^{p_{2}+1}\right) d x .
\end{aligned}
$$

Sobolev embedding theorem, Hölder and Young inequalities imply that

$$
\begin{aligned}
J(u) & \geq\|u\|^{2}-t\|u\|^{2}-C\|u\|^{p_{2}+1} \\
& =(1-t)\|u\|^{2}-C\|u\|^{p_{2}+1} .
\end{aligned}
$$

Finally, if $u \in \mathcal{N}, J(u)=0$ holds and it follows that

$$
\|u\|^{p_{2}-1}=\frac{\|u\|^{p_{2}+1}}{\|u\|^{2}} \geq \frac{1-t}{C} .
$$

b) Note that by (2.2), $\mathcal{N} \cap\{0\}=J^{-1}(\{0\})$. Since $J$ is continuous, this implies that $\mathcal{N}$ is a closed submanifold in $H^{1}\left(\mathbb{R}^{N}\right)$. In addition, if $u \in \mathcal{N}$, then

$$
\begin{aligned}
J^{\prime}(u) u & =2 \int_{\mathbb{R}^{N}}(1+a(x)) f(u) u d x-\int_{\mathbb{R}^{N}}(1+a(x))\left[f^{\prime}(u) u+f(u)\right] u d x \\
& =\int_{\mathbb{R}^{N}}(1+a(x))\left[\frac{f(u)-f^{\prime}(u) u}{u}\right] u^{2} d x<0,
\end{aligned}
$$


as a result of $\left(f_{2}\right)$. Since $f \in C^{1}$, then $J$ is of class $C^{1}$ and hence $\mathcal{N}$ is a $C^{1}$-submanifold.

c) Define the sets

$$
\Gamma^{+}:=\left\{x \in \mathbb{R}^{N}: u(x)>0\right\} \quad \text { and } \quad \Gamma^{-}:=\left\{x \in \mathbb{R}^{N}: u(x)<0\right\} .
$$

If $g(t)=I(t u)$, then

$$
\begin{aligned}
g^{\prime}(t)=t & \|u\|^{2}-\int_{\mathbb{R}^{N}}(1+a(x)) f(t u) u d x \\
=t & \int_{\mathbb{R}^{N}}(1+a(x))\left(\frac{f(u)}{u}-\frac{f(t u)}{t u}\right) u^{2} d x \\
=t & \left\{\int_{\Gamma^{+}}(1+a(x))\left(\frac{f(u)}{u}-\frac{f(t u)}{t u}\right) u^{2} d x\right\} \\
+ & t\left\{\int_{\Gamma^{-}}(1+a(x))\left(\frac{f(u)}{u}-\frac{f(t u)}{t u}\right) u^{2} d x\right\} .
\end{aligned}
$$

By assumption $\left(f_{2}\right)$ we have that $f(s) / s$ is strictly increasing for $s \in(0, \infty)$ and strictly decreasing for $s \in(-\infty, 0)$. Thus, $g^{\prime}(t)>0$, if $t \in(0,1)$ and $g^{\prime}(t)<0$, if $t \in(1, \infty)$. In particular, for all $u \in \mathcal{N}$, we have

$$
g(1)=I(u)=\max _{t>0} g(t)=\max _{t>0} I(t u) .
$$

Thus, we complete the proof.

The following preliminary results are going to be used later and are analogous to those found in [12] under minor modifications. We include them here for the sake of completeness.

Lemma 2.2. Suppose there is a sequence $\left(u_{n}\right)$ in $\mathcal{N}$ satisfying $I\left(u_{n}\right) \rightarrow d$. Then the sequence $\left(u_{n}\right)$ is bounded in $H^{1}\left(\mathbb{R}^{N}\right)$.

Proof. The proof is the same of Lemma 2.2 in [12] using hypotheses $\left(f_{5}\right)$ and $a(\cdot) \in$ $L^{\infty}\left(\mathbb{R}^{N}\right)$.

The following lemma is a result of positivity of the ground state level.

Lemma 2.3. Let $m$ be as in (2.4). Then, it holds that $m>0$.

Proof. The proof is the same of Lemma 2.3 in [12], since $a(\cdot) \in L^{\infty}\left(\mathbb{R}^{N}\right)$.

Lemma 2.4. If $u$ is a solution of problem $\left(P_{a}\right)$ whose associated functional I satisfies $I(u) \in$ $[m, 2 m)$. Then $u$ does not change sign. 
Proof. If $u$ is a solution of $\left(P_{a}\right)$, then $I^{\prime}(u)=0$, i.e., $I^{\prime}(u) v=0$ for all $v \in H^{1}\left(\mathbb{R}^{N}\right)$.

In particular, we have $I^{\prime}(u) u^{+}=0$ and $I^{\prime}(u) u^{-}=0$, where $u^{+}=\max \{u, 0\}$ and $u^{-}=\min \{u, 0\}$. This implies that

$$
\left\langle u, u^{+}\right\rangle-\left\{\int_{\{u \geq 0\}}(1+a(x)) f\left(u^{+}\right) u^{+} d x\right\}=0 .
$$

Thus, $I^{\prime}\left(u^{+}\right) u^{+}=0$ and, similarly, $I^{\prime}\left(u^{-}\right) u^{-}=0$. If $u^{+} \neq 0$ and $u^{-} \neq 0$, then $u^{+}$and $u^{-}$ belong to $\mathcal{N}$. Note that

$$
\begin{aligned}
I(u)= & \frac{1}{2}\left(\left\|u^{+}\right\|^{2}+\left\|u^{-}\right\|^{2}\right)-\int_{\mathbb{R}^{N}}(1+a(x)) F\left(u^{+}\right) d x \\
& \quad-\int_{\mathbb{R}^{N}}(1+a(x)) F\left(u^{-}\right) d x \\
= & I\left(u^{+}\right)+I\left(u^{-}\right) \\
\geq & 2 m .
\end{aligned}
$$

Since $I(u)<2 m$ and $m>0$, by Lemma 2.3, we have a contradiction. This proves the lemma.

Remark 2.1. Lemma 2.4 applies similarly to functional $I_{\infty}$, i.e., if $u$ is the solution of problem $\left(P_{\infty}\right)$ whose associated functional $I_{\infty}$ satisfies $I_{\infty}(u) \in\left[m_{\infty}, 2 m_{\infty}\right)$, then $u$ does not change sign.

A sequence $\left(u_{n}\right)$ in $H^{1}\left(\mathbb{R}^{N}\right)$ is a Palais-Smale sequence at level $c,(P S)_{c}$ for some constant $c$, if $I\left(u_{n}\right) \rightarrow c$ and $I^{\prime}\left(u_{n}\right) \rightarrow 0$ in $H^{-1}\left(\mathbb{R}^{N}\right)$, as $n \rightarrow+\infty$. If a $(P S)_{c}$ sequence $\left(u_{n}\right)$ has a convergent subsequence, we say that $I$ satisfies $(P S)_{c}$ on level $c$.

The next result gives an important information about a $(P S)_{c}$ sequence of $I$ restricted to $\mathcal{N}$.

Lemma 2.5. Assume that $\left(u_{n}\right)$ is a $(P S)_{c}$ sequence for I restricted to $\mathcal{N}$. Then, up to a subsequence, $\left(u_{n}\right)$ is also a $(P S)_{c}$ sequence for the functional $\operatorname{I}$ in $H^{1}\left(\mathbb{R}^{N}\right)$.

Proof. Since $1+a$ is bounded and bounded away from zero, the proof follows as that of Lemma 2.5 in [12], using the theory of constrained critical points in [3].

The classical results concerning the limit problem $\left(P_{\infty}\right)$ found in $[8,9,15]$ together with the study of radial symmetry and exponenctial decay may be summarized as follows: it has a ground state solution $w \in C^{2}\left(\mathbb{R}^{N}\right)$ such that $w>0$ on $\mathbb{R}^{N}, w$ is radially symmetric $(w(x)=w(r)$, where $r=|x|)$ and $w$ decreases with respect to $r$. There exist $C_{1}>0$ and $C_{2}>0$ satisfying

$$
C_{1}(1+|x|)^{-\frac{N-1}{2}} e^{-|x|} \leq w(x) \leq C_{2}(1+|x|)^{-\frac{N-1}{2}} e^{-|x|}, \quad \forall x \in \mathbb{R}^{N} .
$$

For details on uniqueness of the solution $w$ see $[16,23]$. 
Lemma 2.6. There is no solution $u$ for the problem $\left(P_{\infty}\right)$ such that $I_{\infty}(u) \in\left(m_{\infty}, 2 m_{\infty}\right)$.

Proof. Arguing by contradiction, assume that $u$ is solution by $\left(P_{\infty}\right)$ such that $I_{\infty}(u) \in$ $\left(m_{\infty}, 2 m_{\infty}\right)$, then by Remark 2.1, we have $u$ does not change sign. Then, if $u>0$ (the other case is similar), since $\left(P_{\infty}\right)$ has a unique positive radial solution $w$ (see $\left.[21,23]\right)$, it holds that $u=w$ and $I_{\infty}(u)=m_{\infty}$, giving a contradiction.

\section{Compactness and projections on nehari manifold}

The next result describes how a Palais-Smale sequence behaves asymptotically (see [7]).

Lemma 3.1 (Splitting Lemma). Let $\left(u_{n}\right)$ be a bounded sequence in $H^{1}\left(\mathbb{R}^{N}\right)$ such that

$$
I\left(u_{n}\right) \rightarrow d>0 \quad \text { and }\left.\quad I^{\prime}\right|_{\mathcal{N}}\left(u_{n}\right) \rightarrow 0 \quad \text { in } H^{-1}\left(\mathbb{R}^{N}\right) .
$$

Then, up to a subsequence, there exist a solution $u_{0}$ of $(P)$, a number $k \in \mathbb{N} \cup\{0\}$, functions $w^{1}, \cdots, w^{k} \in H^{1}\left(\mathbb{R}^{N}\right)$ solutions of $\left(P_{\infty}\right)$ and sequences of points $\left(y_{n}^{j}\right), 1 \leq j \leq k$, satisfying as $n \rightarrow \infty$,

1. $\left|y_{n}^{j}\right| \rightarrow+\infty$ and $\left|y_{n}^{j}-y_{n}^{i}\right| \rightarrow+\infty ; j \neq i$;

2. $u_{n}-\sum_{i=1}^{k} w^{i}\left(\cdot-y_{n}^{i}\right) \rightarrow u_{0}$ in $H^{1}\left(\mathbb{R}^{N}\right)$;

3. $I\left(u_{n}\right) \rightarrow d=I\left(u_{0}\right)+\sum_{i=1}^{k} I_{\infty}\left(w^{i}\right)$.

Proof. The first step of the proof is Lemma 2.5. The rest of the proof is in $[7,20]$.

Lemma 3.2. Assume that $m$ defined in (2.4) is not attained, then $m \geq m_{\infty}$ and the functional I satisfies Palais-Smale condition on $\mathcal{N}$ at every level $d$ in $\left(m_{\infty}, 2 m_{\infty}\right)$.

Proof. Let $\left(u_{n}\right)$ be a sequence satisfying $(P S)_{d}$ for $I$ restricted to $\mathcal{N}$. Then, up to a subsequence, $\left(u_{n}\right)$ is a bounded $(P S)_{d}$ sequence for $I$ in the function space $H^{1}\left(\mathbb{R}^{N}\right)$ by Lemmas 2.2 and 2.5. If $m$ is not attained, by Lemma 3.1, it holds $m \geq m_{\infty}$. Suppose that $m_{\infty}<d<2 m_{\infty}$ and $\left(u_{n}\right)$ does not have a convergent subsequence, then $k=1$ in Lemma 3.1 and there exists a solution $w^{1}$ of $\left(P_{\infty}\right)$ with $d=I_{\infty}\left(w^{1}\right)$, contradicting Lemma 2.6. Thus, $\left(u_{n}\right)$ has a convergent subsequence and I satisfies the Palais-Smale condition.

In addition, we are going to need the following technical result (see [1]).

Lemma 3.3. Assume that $\mu_{2}>\mu_{1} \geq 0$. Then, there exist a number $C>0$ such that, for all $x_{1}, x_{2} \in \mathbb{R}^{N}$,

$$
\int_{\mathbb{R}^{N}} e^{-\mu_{1}\left|x-x_{1}\right|} e^{-\mu_{2}\left|x-x_{2}\right|} d x \leq C e^{-\mu_{1}\left|x_{1}-x_{2}\right|} .
$$


If $\mu_{2} \geq \mu_{1}>0$ and $\mu_{3}>\mu_{1} \geq 0$. Then, there exist a number $C>0$ such that, for all $x_{1}, x_{2}, x_{3} \in \mathbb{R}^{N}$, it holds

$$
\int_{\mathbb{R}^{N}} e^{-\mu_{1}\left|x-x_{1}\right|} e^{-\mu_{2}\left|x-x_{2}\right|} e^{-\mu_{3}\left|x-x_{3}\right|} d x \leq C e^{-\frac{\mu_{1}}{2}\left(\left|x_{1}-x_{2}\right|+\left|x_{1}-x_{3}\right|+\left|x_{2}-x_{3}\right|\right)} .
$$

Now, we are going to show that $\mathcal{N}$ is not empty and also study the projections on $\mathcal{N}$.

Remark 3.1. Assume two real number $r \in(0,+\infty)$ and $\lambda \in[0,1]$ and let us define a function

$$
\Phi(\lambda, r):=\lambda^{2}\left(\|w\|^{2}-\int_{\mathbb{R}^{N}} \frac{f(r \lambda w)}{r \lambda w} w^{2} d x\right)
$$

where $w$ is the ground state solution of $\left(P_{\infty}\right)$. Let us consider the autonomous limit problem $\left(P_{\infty}\right)$, then it follows from [12] (see Lemma 3.1) that there exist $S_{0}<0$ and $T_{0}>0$ such that for all $r \geq T_{0}$ it holds

$$
\Phi(\lambda, r)+\Phi(1-\lambda, r) \leq S_{0}<0, \quad \forall \lambda \in[0,1] .
$$

Let us fix $y_{0} \in \mathbb{R}^{N},\left|y_{0}\right|=1$, and take $y \in \partial B_{2}\left(y_{0}\right)$, where

$$
B_{2}\left(y_{0}\right):=\left\{x \in \mathbb{R}^{N} ;\left|x-y_{0}\right| \leq 2\right\} .
$$

For $w$ the solution of $\left(P_{\infty}\right)$, we will define

$$
w_{0}^{R}:=w\left(x-R y_{0}\right) \quad \text { and } \quad w_{y}^{R}:=w(x-R y),
$$

and consider a linear combination for any $\lambda \in[0,1]$

$$
\begin{aligned}
z^{R} & =z_{\lambda, y}^{R}:=\lambda w\left(x-R y_{0}\right)+(1-\lambda) w(x-R y) \\
& =\lambda w_{0}^{R}+(1-\lambda) w_{y}^{R} .
\end{aligned}
$$

The next lemma follows closely Lemma 3.2 in [12].

Lemma 3.4. i) There exist $R_{0}>0, T_{0}>2$ and for each $R \geq R_{0}, y \in \partial B_{2}\left(y_{0}\right)$ and $0 \leq \lambda \leq 1$, a unique $T_{\lambda, y}^{R}>0$ satisfying $T_{\lambda, y}^{R} z^{R} \in \mathcal{N}$. Furthermore, $T_{\lambda, y}^{R} \in\left(0, T_{0}\right)$ and $T_{\lambda, y}^{R}$ is a continuous function of the variables $\lambda, y, R$;

ii) Consider $\lambda=\frac{1}{2}$. If $R \rightarrow+\infty$, then $T_{\lambda, y}^{R} \rightarrow 2$, uniformly in $y \in \partial B_{2}\left(y_{0}\right)$.

Proof. i) If $u, v \in H^{1}\left(\mathbb{R}^{N}\right), u, v>0$ and $r \in(0,+\infty)$, then

$$
\begin{aligned}
& J_{\infty}(r u+r v)=I_{\infty}^{\prime}(r u+r v)(r u+r v) \\
= & r^{2}\left(\|u\|^{2}+\|v\|^{2}+2\langle u, v\rangle\right)-r^{2} \int_{\mathbb{R}^{N}} \frac{f(r u+r v)}{r u+r v}\left(u^{2}+2 u v+v^{2}\right) d x .
\end{aligned}
$$


$\operatorname{Using}\left(f_{2}\right)$,

$$
\frac{J_{\infty}(r u+r v)}{r^{2}}<\|u\|^{2}-\int_{\mathbb{R}^{N}} \frac{f(r u)}{r u}\left(u^{2}\right) d x+\|v\|^{2}-\int_{\mathbb{R}^{N}} \frac{f(r v)}{r v}\left(v^{2}\right) d x+2\langle u, v\rangle .
$$

In (3.2) write $u:=\lambda w_{0}^{R}$ and $v:=(1-\lambda) w_{y}^{R}$,

$$
\begin{aligned}
& \frac{J_{\infty}(r u+r v)}{r^{2}}=\frac{J_{\infty}\left(r \lambda w_{0}^{R}+r(1-\lambda) w_{y}^{R}\right)}{r^{2}} \\
& \leq \lambda^{2}\left(\|w\|^{2}-\int_{\mathbb{R}^{N}} \frac{f(r \lambda w)}{r \lambda w}(w)^{2} d x\right)+(1-\lambda)^{2}\left(\|w\|^{2}-\int_{\mathbb{R}^{N}} \frac{f(r(1-\lambda) w)}{r(1-\lambda) w}(w)^{2} d x\right) \\
& \quad+2 \lambda(1-\lambda)\left\langle w_{0}^{R}, w_{y}^{R}\right\rangle .
\end{aligned}
$$

By Remark 3.1, the decay estimates (2.7) and Lemma 3.3 it follows

$$
\begin{aligned}
& \frac{J_{\infty}\left(r \lambda w_{0}^{R}+r(1-\lambda) w_{y}^{R}\right)}{r^{2}} \\
\leq & \Phi(\lambda, r)+\Phi(1-\lambda, r)+o_{R}(1)<S_{0}+o_{R}(1) .
\end{aligned}
$$

Moreover, by the definitions of $J$ and $J_{\infty}$,

$$
\begin{aligned}
& \frac{J\left(r \lambda w_{0}^{R}+r(1-\lambda) w_{y}^{R}\right)}{r^{2}} \\
= & \frac{J_{\infty}\left(r \lambda w_{0}^{R}+r(1-\lambda) w_{y}^{R}\right)}{r^{2}} \\
& \quad-\int_{\mathbb{R}^{N}} a(x) f\left(r \lambda w_{0}^{R}+r(1-\lambda) w_{y}^{R}\right)\left(\lambda w_{0}^{R}+(1-\lambda) w_{y}^{R}\right) d x .
\end{aligned}
$$

On the other hand, by $\left(a_{2}\right),\left(f_{1}\right)$ and Lemma 3.3 it follows

$$
\int_{\mathbb{R}^{N}} a(x) f\left(r \lambda w_{0}^{R}+r(1-\lambda) w_{y}^{R}\right)\left(r \lambda w_{0}^{R}+r(1-\lambda) w_{y}^{R}\right) d x=o_{R}(1),
$$

since

$$
\begin{aligned}
& \int_{\mathbb{R}^{N}} a(x)\left(w_{0}^{R}\right)^{p_{i}+1} d x \leq C \int_{\mathbb{R}^{N}} e^{-\kappa|x|} e^{-\left(p_{i}+1\right)\left|x-R y_{0}\right|}=o_{R}(1), \\
& \int_{\mathbb{R}^{N}} a(x)\left(w_{y}^{R}\right)^{p_{i}+1} d x \leq C \int_{\mathbb{R}^{N}} e^{-\kappa|x|} e^{-\left(p_{i}+1\right)|x-R y|}=o_{R}(1),
\end{aligned}
$$

for $i=1,2$, and $\left\langle w_{0}^{R}, w_{y}^{R}\right\rangle=o_{R}(1)$. Combining (3.4), (3.6) with (3.5), it results

$$
\frac{J\left(r \lambda w_{0}^{R}+r(1-\lambda) w_{y}^{R}\right)}{r^{2}} \leq S_{0}+o_{R}(1)<\frac{S_{0}}{3}<0, \quad \forall r \geq T_{0},
$$


$0 \leq \lambda \leq 1$ and uniformily in $y \in \partial B_{2}\left(y_{0}\right)$. By (3.7) and Lemma 2.1, there exists $T_{\lambda, y}^{R} \in$ $\left(0, T_{0}\right)$ such that $J\left(T_{\lambda, y}^{R} z_{\lambda, y}^{R}\right)=0$ and this concludes part i).

ii) Definition (3.1) with $\lambda=\frac{1}{2}$ gives

$$
z^{R}=\frac{1}{2} w\left(x-R y_{0}\right)+\frac{1}{2} w(x-R y)=\frac{1}{2} w_{0}^{R}+\frac{1}{2} w_{y}^{R} .
$$

Substituting in (2.3), it holds

$$
\begin{array}{r}
\left.J\left(2 z^{R}\right) \leq\left\|w_{0}^{R}\right\|^{2}+\left\|w_{y}^{R}\right\|^{2}-\int_{\mathbb{R}^{N}} f\left(w_{0}^{R}\right) w_{0}^{R}\right) d x-\int_{\mathbb{R}^{N}} f\left(w_{y}^{R}\right) w_{y}^{R} d x \\
+2\left\langle w_{0}^{R}, w_{y}^{R}\right\rangle-\int_{\mathbb{R}^{N}} a(x)\left[f\left(w_{0}^{R}\right) w_{0}^{R}+f\left(w_{y}^{R}\right) w_{y}^{R}\right] d x .
\end{array}
$$

Assumptions $\left(a_{2}\right),\left(f_{1}\right)$ and Lemma 3.3 yield

$$
\begin{aligned}
& \left|\int_{\mathbb{R}^{N}} a(x) f\left(w_{0}^{R}\right) w_{0}^{R} d x\right| \\
\leq & C \int_{\mathbb{R}^{N}} e^{-\kappa|x|}\left[\left|w_{0}^{R}\right|^{p_{1}+1}+\left|w_{0}^{R}\right|^{p_{2}+1}\right] d x=C e^{-\kappa R}=o_{R}(1) .
\end{aligned}
$$

Since $w$ is a solution of $\left(P_{\infty}\right)$, i.e.,

$$
J_{\infty}\left(w_{0}^{R}\right)=J_{\infty}\left(w_{y}^{R}\right)=J_{\infty}(w)=0 \quad \text { and }\left\langle w_{0}^{R}, w_{y}^{R}\right\rangle=o_{R}(1),
$$

we obtain $(3.8) \leq o_{R}(1)$. This proves ii).

\section{Asymptotic estimates}

The next result is decisive in order to estimate the energy levels of critical points. As opposed to [14], where the condition $f(u)=o\left(|u|^{1+v}\right)$, as $|u| \rightarrow 0$ was assumed, here we are going to work under hypothesis $\left(f_{1}\right)$, for instance, see Remark 1.1.

Lemma 4.1. Assume $\left(f_{1}\right)$ and $\left(f_{2}\right)$. If $u, v \in \mathbb{R}$, then

$$
F(u+v) \geq F(u)+f(u) v,
$$

and if $0 \leq u, v \leq \rho$, then there exists a constant $C_{\rho} \geq 0$ such that

$$
F(u+v)-F(u)-F(v) \geq f(u) v+f(v) u-C_{\rho} u^{1+\frac{\mu}{2}} v^{1+\frac{\mu}{2}} .
$$

Proof. A consequence of $\left(f_{2}\right)$ is that the function $u \mapsto f(u)$ is increasing, which yields

$$
F(u+v)-F(u)=\int_{u}^{u+v} f(t) d t \geq f(u) v
$$


Furthermore, if $u=0$ or $v=0,(4.2)$ is obvious. In case $0<v \leq u$, we deduce from (4.1) and $\left(f_{1}\right)$ (see Remark (1.1))

$$
\begin{aligned}
& F(u+v)-F(u)-F(v)-f(u) v-f(v) u \\
\geq & -F(v)-f(v) u \\
= & -\int_{0}^{v} \frac{f(t)}{t^{1+\mu}} t^{1+\mu} d t-\frac{f(v)}{v^{1+\mu}} u v^{1+\mu} \\
\geq & -\frac{\bar{C}_{\rho}}{2+\mu} v^{2+\mu}-\bar{C}_{\rho} u v^{1+\mu} \\
= & -\bar{C}_{\rho}(u v)^{1+\frac{\mu}{2}}\left\{\frac{1}{2+\mu}+1\right\} \\
\geq & -\frac{3}{2} \bar{C}_{\rho} u^{1+\frac{\mu}{2}} v^{1+\frac{\mu}{2}}
\end{aligned}
$$

where

$$
\bar{C}_{\rho}:=\sup _{0<u \leq \rho} \frac{f(u)}{u^{1+\mu}}<\infty
$$

and for every $\mu>0$ satisfying $0<1+\mu<p_{1}$. Since (4.1) and (4.2) are symmetric in $u$ and $v$, the same estimate holds for $0<u \leq v$, and the proof is completed.

Let us now obtain further precise estimates. In order to do so, as in the literature, $y \in \partial B_{2}\left(y_{0}\right), y_{0}$ is fixed satisfying $\left|y_{0}\right|=1$, for $R>0$, we define the quantity

$$
\varepsilon_{R}:=\int_{\mathbb{R}^{N}} f\left(w\left(x-R y_{0}\right)\right) w(x-R y) d x,
$$

where $w$ is the positive radial ground state solution of $\left(P_{\infty}\right)$. For additional information about (4.3), see $[1,4,11,12,19]$ and references therein.

The next result of Bahri and Lions is essential for proving the asymptotic behaviour of quantity $\varepsilon_{R}$.

Lemma 4.2. Let $\varphi \in C\left(\mathbb{R}^{N}\right) \cap L^{\infty}\left(\mathbb{R}^{N}\right), \psi \in C\left(\mathbb{R}^{N}\right)$ satisfy for some $\alpha, \beta \geq 0$ and $\gamma \in \mathbb{R}$,

$$
\begin{gathered}
\varphi(x) e^{\alpha|x|}|x|^{\beta} \rightarrow \gamma, \quad \text { if }|x| \rightarrow+\infty, \\
\int_{\mathbb{R}^{N}}|\psi(x)| e^{\alpha|x|}\left(1+|x|^{\beta}\right) d x<+\infty .
\end{gathered}
$$

Then

$$
\lim _{|y| \rightarrow+\infty}\left[\left(\int_{\mathbb{R}^{N}} \varphi(x+y) \psi(x) d x\right) e^{\alpha|y|}|y|^{\beta}-\gamma \int_{\mathbb{R}^{N}} e^{-\frac{\alpha\langle x, y\rangle}{|y|}} \psi(x) d x\right]=0 .
$$

Proof. See [4] (see Lemma II.2). 
Lemma 4.3. Assume $\left(f_{1}\right)$ and let $y \in \partial B_{2}\left(y_{0}\right)$ with $y_{0} \in \mathbb{R}^{N},\left|y_{0}\right|=1$. Then, there exists a constante $C_{0}>0$ such that

$$
\lim _{R \rightarrow+\infty} \varepsilon_{R}(2 R)^{\frac{N-1}{2}} e^{2 R}=C_{0} .
$$

Proof. We use Lemma 4.2 with $\varphi=w, \psi=f(w)$ and $z=-R\left(y_{0}-y\right)$ and with $\alpha=1$ and $\beta=\frac{N-1}{2}$. From (2.7), it holds

$$
\lim _{|x| \rightarrow \infty} w(x)|x|^{\frac{N-1}{2}} e^{|x|}=\sigma>0 .
$$

On the other hand, using $\left(f_{1}\right)$ and (4.7) there exists $R_{1}>0$ such that, for all $|x|>R_{1}$,

$$
\begin{aligned}
\psi & =f(w) \leq C\left(|w|^{p_{1}}+|w|^{p_{2}}\right) \\
& \leq C\left(|x|^{-p_{1} \frac{N-1}{2}} e^{-p_{1}|x|}+|x|^{-p_{2} \frac{N-1}{2}} e^{-p_{2}|x|}\right),
\end{aligned}
$$

which yields

$$
\begin{aligned}
& \int_{\mathbb{R}^{N}} f(w) e^{|x|}(1+|x|)^{\frac{N-1}{2}} d x \\
= & \int_{B_{R_{1}}(0)} f(w) e^{|x|}(1+|x|)^{\frac{N-1}{2}} d x+\int_{\mathbb{R}^{N} \backslash B_{R_{1}}(0)} f(w) e^{|x|}(1+|x|)^{\frac{N-1}{2}} d x \\
\leq & C \mu\left(B_{R_{1}}(0)\right)+\int_{\mathbb{R}^{N} \backslash B_{R_{1}}(0)} f(w) e^{|x|}(1+|x|)^{\frac{N-1}{2}} d x \\
= & C+C \int_{\mathbb{R}^{N} \backslash B_{R_{1}}(0)} e^{\left(1-p_{1}\right)|x|}(1+|x|)^{\frac{N-1}{2}} d x \\
& +C \int_{\mathbb{R}^{N} \backslash B_{R_{1}}(0)} e^{\left(1-p_{2}\right)|x|}(1+|x|)^{\frac{N-1}{2}} d x<+\infty,
\end{aligned}
$$

since $1-p_{i}<0$ for $i \in\{1,2\}$. Using Lemma 4.2, we obtain

$$
\lim _{R \rightarrow+\infty} \varepsilon_{R}(2 R)^{\frac{N-1}{2}} e^{2 R}=C_{0}>0 .
$$

Thus, we complete the proof.

On the other hand, an inferior bound for $\varepsilon_{R}$ may be obtained.

Lemma 4.4. There exists a constant $C_{3}>0$ such that

$$
\int_{\mathbb{R}^{N}} f\left(s w\left(x-R y_{0}\right)\right) t w(x-R y) d x \geq C_{3}(2 R)^{-\frac{N-1}{2}} e^{-2 R},
$$

with $y \in \partial B_{2}\left(y_{0}\right)$ and for all $t, s \geq 1 / 2$. 
Proof. First, for $s, t \geq 1 / 2$ and from $\left(f_{2}\right)$

$$
\begin{aligned}
\int_{R^{N}} f\left(s w_{0}\right) t w_{y} d x & \geq \frac{1}{4} \int_{B_{1}\left(R y_{0}\right)} \frac{f\left(\frac{1}{2} w_{0}\right)}{\frac{1}{2} w_{0}} w_{0} w_{y} d x \\
& \geq \frac{1}{4} \min _{B_{1}(0)} \frac{f\left(\frac{1}{2} w(x)\right)}{\frac{1}{2} w(x)} \int_{B_{1}(0)} w(x) w\left(x-R\left(y-y_{0}\right)\right) d x \\
& \geq \frac{1}{4} C(2 R)^{-\frac{N-1}{2}} e^{-2 R},
\end{aligned}
$$

since for $x \in B_{1}(0)$ and $R \geq 1$ it holds

$$
1+\left|x-R\left(y-y_{0}\right)\right| \leq 1+|x|+R\left|y-y_{0}\right| \leq R+R+2 R=4 R
$$

and from (4.8) and (2.7) it follows

$$
\begin{aligned}
w\left(x-R\left(y-y_{0}\right)\right) & \geq C_{1}\left(1+\left|x-R\left(y-y_{0}\right)\right|\right)^{-\frac{N-1}{2}} e^{-\mid x-R\left(y-y_{0} \mid\right.} \\
& \geq C(2 R)^{-\frac{N-1}{2}} e^{-2 R} .
\end{aligned}
$$

The proof of the lemma is completed.

The following result presents some crucial estimate if $s$ approaches 1 .

Corollary 4.1. Given $b>0$, there exists a positive constant $C$, such that

$$
\left|\int_{R^{N}}\left(s f\left(w_{0}^{R}\right)-f\left(s w_{0}^{R}\right)\right) w_{y}^{R} d x\right| \leq C|s-1| \mathcal{O}\left(\varepsilon_{R}\right),
$$

uniformly in $y \in \partial B_{2}\left(y_{0}\right), s \in[0, b]$ and $R$ large enough.

Proof. Consider the function $\psi(s):=s f(u)-f(s u)$. The mean value theorem implies that there exists $\xi$ between $s$ and 1, without loss of generality $s>1$, such that

$$
|\psi(s)-\psi(1)| \leq\left|\psi^{\prime}(\xi)\right||s-1| \text {. }
$$

$\operatorname{Using}\left(f_{1}\right)$,

$$
\left|\psi^{\prime}(s)\right|=\left|f(u)-f^{\prime}(s u) u\right| \leq|f(u)|+C\left(|u|^{p_{1}}+|u|^{p_{2}}\right) .
$$

Combining (4.9) and (4.10), it holds

$$
|\psi(s)|=|\psi(s)-\psi(1)| \leq\left(|f(u)|+C\left(|u|^{p_{1}}+|u|^{p_{2}}\right)\right)|s-1| .
$$

Taking $u=w_{0}^{R}$, then

$$
\begin{aligned}
& \left|\int_{\mathbb{R}^{N}}\left(s f\left(w_{0}^{R}\right)-f\left(s w_{0}^{R}\right)\right) w_{y}^{R} d x\right| \\
\leq & |s-1|\left(\int_{\mathbb{R}^{N}}\left|f\left(w_{0}^{R}\right) w_{y}^{R}\right|+C \int_{\mathbb{R}^{N}}\left(\left|w_{0}^{R}\right|^{p_{1}} w_{y}^{R}+\left|w_{0}^{R}\right|^{p_{2}} w_{y}^{R}\right) d x\right) \\
\leq & |s-1|\left(\varepsilon_{R}+\mathcal{O}\left(\varepsilon_{R}\right)\right),
\end{aligned}
$$


and we may use (2.7), Lemma 3.3 and argue as in the proof of Lemma 4.3 to obtain

$$
\int_{\mathbb{R}^{N}}\left|w_{0}^{R}\right|^{p_{i}} w_{y}^{R} d x \leq \mathcal{O}\left(\varepsilon_{R}\right) .
$$

This proves the corollary.

Now, we are ready to show an important estimate for the energy functional $I$ which will play a crucial role in the proof of the main result.

Lemma 4.5. There exist numbers $R_{2}>0$ and for each $R>R_{2}$, a number $\alpha=\alpha_{R}>0$ such that

$$
I\left(T_{\lambda, y}^{R} z_{\lambda, y}^{R}\right) \leq 2 m_{\infty}-\alpha
$$

for all $y \in \partial B_{2}\left(y_{0}\right)$ and all $\lambda \in[0,1]$.

Proof. First of all, we write

$$
\lambda T_{\lambda, y}^{R} w\left(x-R y_{0}\right)+(1-\lambda) T_{\lambda, y}^{R} w(x-R y):=s w_{0}^{R}+t w_{y}^{R} .
$$

By Lemma 3.4, we know that $s, t \in\left(0, T_{0}\right)$ and

$$
\begin{aligned}
& I\left(s w_{0}^{R}+t w_{y}^{R}\right) \\
=\frac{1}{2} \int_{\mathbb{R}^{N}}\left(s^{2}\left|\nabla w_{0}^{R}\right|^{2}+t^{2}\left|\nabla w_{y}^{R}\right|^{2}+2 s t \nabla w_{0}^{R} \nabla w_{y}^{R}\right) d x & \\
& +\frac{1}{2} \int_{\mathbb{R}^{N}}\left(s^{2}\left|w_{0}^{R}\right|^{2}+t^{2}\left|w_{y}^{R}\right|^{2}+2 s t w_{0}^{R} w_{y}^{R}\right) d x \\
& -\int_{\mathbb{R}^{N}}(1+a(x)) F\left(s w_{0}^{R}+t w_{y}^{R}\right) d x \\
=\frac{s^{2}}{2} & \int_{\mathbb{R}^{N}}\left(|\nabla w|^{R}+\left(w_{0}^{R}\right)^{2}\right) d x+\frac{t^{2}}{2} \int_{\mathbb{R}^{N}}\left(\left|\nabla w_{y}^{R}\right|^{2}+\left(w_{y}^{R}\right)^{2}\right) d x \\
& -\int_{\mathbb{R}^{N}} F\left(s w_{0}^{R}\right) d x-\int_{\mathbb{R}^{N}} F\left(t w_{y}^{R}\right) d x \\
& -\int_{\mathbb{R}^{N}}\left[F\left(s w_{0}^{R}+t w_{y}^{R}\right)-F\left(s w_{0}^{R}\right)-F\left(t w_{y}^{R}\right)\right] d x \\
& +s t \int_{\mathbb{R}^{N}}\left(\nabla w_{0}^{R} \nabla w_{y}^{R}+w_{0}^{R} w_{y}^{R}\right) d x-\int_{\mathbb{R}^{N}}\left(f\left(s w_{0}^{R}\right) t w_{y}^{R}+f\left(t w_{y}^{R}\right) s w_{0}^{R}\right) d x \\
& +\int_{\mathbb{R}^{N}}\left(f\left(s w_{0}^{R}\right) t w_{y}^{R}+f\left(t w_{y}^{R}\right) s w_{0}\right) d x \\
& -\int_{\mathbb{R}^{N}} a(x) F\left(s w_{0}^{R}+t w_{y}^{R}\right) d x .
\end{aligned}
$$


Let us estimate every line in the equation above. In the lines $(A)$ and $(B)$, as $w_{0}^{R}$ and $w_{y}^{R}$ are least energy solutions of the problem $\left(P_{\infty}\right)$,

$$
\begin{aligned}
& \frac{s^{2}}{2} \int_{\mathbb{R}^{N}}\left(\left|\nabla w_{0}^{R}\right|^{2}+\left(w_{0}^{R}\right)^{2}\right) d x-\int_{\mathbb{R}^{N}} F\left(s w_{0}^{R}\right) d x=I_{\infty}\left(s w_{0}^{R}\right) \leq m_{\infty}, \\
& \frac{t^{2}}{2} \int_{\mathbb{R}^{N}}\left(\left|\nabla w_{0}^{R}\right|^{2}+\left(w_{0}^{R}\right)^{2}\right) d x-\int_{\mathbb{R}^{N}} F\left(t w_{0}^{R}\right) d x=I_{\infty}\left(t w_{0}^{R}\right) \leq m_{\infty} .
\end{aligned}
$$

To estimate $(C)$, first we use Lemma 4.1 to obtain

$$
\begin{gathered}
\int_{\mathbb{R}^{N}}\left[F\left(s w_{0}^{R}+t w_{y}^{R}\right)-F\left(s w_{0}^{R}\right)-F\left(t w_{y}^{R}\right)\right] d x \\
\geq t \int_{\mathbb{R}^{N}} f\left(s w_{0}^{R}\right) w_{y}^{R} d x+s \int_{R^{N}} f\left(t w_{y}^{R}\right) w_{0}^{R} d x \\
\quad-C_{\rho}(s t)^{1+\frac{\mu}{2}} \int_{\mathbb{R}^{N}}\left(w_{0}^{R}\right)^{1+\frac{\mu}{2}}\left(w_{y}^{R}\right)^{1+\frac{\mu}{2}} d x .
\end{gathered}
$$

Then we take $1<\bar{\mu}<1+\frac{\mu}{2}$ and by Lemma 3.3

$$
\begin{aligned}
& \int_{\mathbb{R}^{N}}\left(w_{0}^{R}\right)^{1+\frac{\mu}{2}}\left(w_{y}^{R}\right)^{1+\frac{\mu}{2}} d x \\
\leq & C \int_{\mathbb{R}^{N}} e^{-\left(1+\frac{\mu}{2}\right)\left|x-R y_{0}\right|} e^{-\left(1+\frac{\mu}{2}\right)|x-R y|} d x \\
\leq & C e^{-\bar{\mu}\left|R y_{0}-R y\right|}=C e^{-2 \bar{\mu} R}=o\left(\varepsilon_{R}\right) .
\end{aligned}
$$

Therefore, (4.13) and (4.14) yield

$$
\begin{aligned}
& -\int_{\mathbb{R}^{N}}\left[F\left(s w_{0}^{R}+t w_{y}^{R}\right)-F\left(s w_{0}^{R}\right)-F\left(t w_{y}^{R}\right)\right] d x \\
& \quad+t \int_{\mathbb{R}^{N}} f\left(s w_{0}^{R}\right) w_{y}^{R} d x+s \int_{\mathbb{R}^{N}} f\left(t w_{y}^{R}\right) w_{0}^{R} d x \leq o\left(\varepsilon_{R}\right),
\end{aligned}
$$

which gives

$$
(C)+(E) \leq o\left(\varepsilon_{R}\right) .
$$

We use Corollary 4.1 and Lemma 4.4 to estimate $(D)$ :

$$
\begin{aligned}
& \quad s t \int_{\mathbb{R}^{N}}\left(\nabla w_{0}^{R} \nabla w_{y}^{R}+w_{0}^{R} w_{y}^{R}\right) d x-\int_{\mathbb{R}^{N}}\left(f\left(s w_{0}^{R}\right) t w_{y}^{R}+f\left(t w_{y}^{R}\right) s w_{0}^{R}\right) d x \\
& =\frac{s t}{2} \int_{\mathbb{R}^{N}}\left(\nabla w_{0}^{R} \nabla w_{y}+w_{0}^{R} w_{y}^{R}\right) d x-\frac{1}{2} \int_{\mathbb{R}^{N}} f\left(s w_{0}^{R}\right) t w_{y}^{R} d x \\
& \quad+\frac{s t}{2} \int_{\mathbb{R}^{N}}\left(\nabla w_{y}^{R} \nabla w_{0}^{R}+w_{y}^{R} w_{0}^{R}\right) d x-\frac{1}{2} \int_{\mathbb{R}^{N}} f\left(t w_{y}^{R}\right) s w_{0}^{R} d x \\
& \quad-\frac{1}{2} \int_{\mathbb{R}^{N}}\left(f\left(s w_{0}^{R}\right) t w_{y}^{R}+f\left(t w_{y}^{R}\right) s w_{0}^{R}\right) d x
\end{aligned}
$$




$$
\begin{aligned}
& =\frac{t}{2} \int_{\mathbb{R}^{N}}\left(s f\left(w_{0}^{R}\right)-f\left(s w_{0}^{R}\right)\right) w_{y}^{R}+\frac{s}{2} \int_{\mathbb{R}^{N}}\left(t f\left(w_{y}^{R}\right)-f\left(t w_{y}^{R}\right)\right) w_{0}^{R} \\
& \quad-\frac{1}{2} \int_{\mathbb{R}^{N}}\left(f\left(s w_{0}^{R}\right) t w_{y}^{R}+f\left(t w_{y}^{R}\right) s w_{0}^{R}\right) d x \\
& \leq C(|s-1|+|t-1|) \mathcal{O}\left(\varepsilon_{R}\right)-C_{0} \varepsilon_{R} .
\end{aligned}
$$

By Lemma 3.4ii), if $\lambda=1 / 2$, then $s, t \rightarrow 1$, as $R \rightarrow+\infty$. Taking $R_{2}$ large enough and $\sigma=\sigma(R) \in(0,1 / 2)$ sufficiently small, it follows by continuity with respect to $\lambda$

$$
(D) \leq-\frac{C_{0}}{2} \varepsilon_{R}
$$

for all

$$
\lambda \in\left[\frac{1}{2}-\sigma, \frac{1}{2}+\sigma\right], \quad y \in \partial B_{2}\left(y_{0}\right), \quad R>R_{2} .
$$

It only remains to estimate $(F)$; by $\left(a_{2}\right)$ and Lemma 3.3,

$$
\begin{aligned}
& \left|\int_{\mathbb{R}^{N}} a(x) F\left(s w w_{0}^{R}+t w_{y}^{R}\right) d x\right| \\
\leq & C \int_{\mathbb{R}^{N}} e^{-k|x|}\left(\left|w_{0}^{R}\right|^{p_{1}+1}+\left|w_{0}^{R}\right|^{p_{2}+1}+\left|w_{y}^{R}\right|^{p_{1}+1}+\left|w_{y}^{R}\right|^{p_{2}+1}\right) d x \\
= & o\left(\varepsilon_{R}\right) .
\end{aligned}
$$

It follows by (4.12a), (4.12b), (4.16), (4.17), (4.18), (4.19) that

$$
\begin{aligned}
I\left(s w w_{0}^{R}+t w w_{y}^{R}\right) & =I\left(\lambda \operatorname{Tw}\left(x-R y_{0}\right)+(1-\lambda)\right) \operatorname{Tw}(x-R y) \\
& \leq 2 m_{\infty}-\frac{C_{0}}{2} \varepsilon_{R}+o\left(\varepsilon_{R}\right) .
\end{aligned}
$$

Furthermore, applying Lemma 2.1, $I_{\infty}\left(t w_{0}^{R}\right)<m_{\infty}-\eta$ for some

$$
\eta \in\left(0, m_{\infty}\right), \quad t \in\left[0,1-\sigma_{0}\right] \cup\left[1+\sigma_{0}, \infty\right) .
$$

Combining this with (4.12a)-(4.19), we obtain

$$
\begin{aligned}
I\left(s w_{0}^{R}+t w_{y}^{R}\right) & =I\left(\lambda T w\left(x-R y_{0}\right)+(1-\lambda) T w(x-R y)\right) \\
& \leq m_{\infty}-\eta+m_{\infty}-\eta+\mathcal{O}\left(\varepsilon_{R}\right),
\end{aligned}
$$

for all

$$
\lambda \in\left[0, \frac{1}{2}-\sigma\right] \cup\left[\frac{1}{2}+\sigma, 1\right] .
$$

Thus, we conclude the proof with (4.20) and (4.21), for all $\lambda \in[0,1], y \in \partial B_{2}\left(y_{0}\right)$ and $R>R_{2}$.

Lemma 4.6. For any $\eta>0$, there exists $R_{3}>0$ such that

$$
I\left(T_{\lambda, y}^{R} z_{y}^{R}\right)<m_{\infty}+\eta,
$$

for all $y \in \partial B_{2}\left(y_{0}\right), R>R_{3}$ and $\lambda=0$. Particulary, $m \leq m_{\infty}$ 
Proof. We already know that, for all $u \in H^{1}\left(\mathbb{R}^{N}\right)$,

$$
I(u)=I_{\infty}(u)-\int_{\mathbb{R}^{N}} a(x) F(u) d x .
$$

Let $\lambda=0$ and $T_{\lambda, y}^{R}$ in (4.11). Using Lemma 2.1 and (4.19) it holds

$$
\begin{aligned}
I\left(T_{0, y}^{R} w_{y}\right) & =I_{\infty}\left(T_{0, y}^{R} w_{y}^{R}\right)-\int_{\mathbb{R}^{N}} a(x) F\left(T_{0, y}^{R} w_{y}^{R}\right) d x \\
& \leq \max _{t>0} I_{\infty}\left(t w_{y}^{R}\right)+\int_{\mathbb{R}^{N}}|a(x)|\left|F\left(T_{0, y}^{R} w_{y}^{R}\right)\right| d x \\
& =m_{\infty}+\int_{\mathbb{R}^{N}}|a(x)|\left|F\left(T_{0, y}^{R} w_{y}^{R}\right)\right| d x \\
& \leq m_{\infty}+o\left(\varepsilon_{R}\right),
\end{aligned}
$$

as claimed.

\section{Topological arguments and proof of main result}

In order to define the linking sets, we will make use of the properties of a barycenter function. Let a function $\beta: H^{1}\left(\mathbb{R}^{N}\right) \backslash\{0\} \rightarrow \mathbb{R}^{N}$. First of all, we define the following $\operatorname{maps} \Psi_{u}, v: \mathbb{R}^{N} \rightarrow \mathbb{R}$ where

$$
\Psi_{u}:=\frac{1}{\mu\left(B_{1}(0)\right)} \int_{B_{1}(x)}|u(y)| d y \quad \text { and } \quad v(x):=\left(\Psi_{u}-\frac{1}{2} \max _{x \in \mathbb{R}^{N}} \Psi_{u}(x)\right)^{+} .
$$

The barycenter function is defined by

$$
\beta(u):=\frac{1}{|v|_{1}} \int_{\mathbb{R}^{N}} x v(x) d x .
$$

The barycenter function has the following properties: $\beta$ is continuous on $H^{1}\left(\mathbb{R}^{N}\right) \backslash\{0\}$; if $u \in H^{1}\left(\mathbb{R}^{N}\right) \backslash\{0\}$ is radial, then $\beta(u)=0$; for every $t \in \mathbb{R} \backslash\{0\}$ and $u \in H^{1}\left(\mathbb{R}^{N}\right) \backslash\{0\}$, $\beta(u)=\beta(t u)$ and if $z \in \mathbb{R}^{N}$ it follows that $\beta(u(x-z))=\beta(u)+z$.

The next lemma and the proof of the main result are very similar to those found in [12], as long as we have gathered all the previous information. We recall them here for the sake of completeness.

Lemma 5.1. Assume that $m$ is not attained. Then $m=m_{\infty}$ and there exists some $\eta>0$ such that

$$
\forall u \in \mathcal{N} \cap I^{m_{\infty}+\eta} ; \quad \beta(u) \neq 0,
$$

where $I^{m_{\infty}+\eta}:=\left\{u \in H^{1}\left(\mathbb{R}^{N}\right) ; I(u) \leq m_{\infty}+\eta\right\}$. 
Proof. Assume that $m$ is not attained. By Lemma 3.2, $m \geq m_{\infty}$ and Lemma 4.6 imply that $m \leq m_{\infty}$. Hence, $m=m_{\infty}$. Assume that for each $n \in \mathbb{N}$, there exists $\left(v_{n}\right) \subset \mathcal{N} \cap I^{m_{\infty}+\alpha}$, i.e., $\left(v_{n}\right) \subset \mathcal{N}$ satisfying $I\left(v_{n}\right)<m_{\infty}+o_{n}(1)$ with $\beta\left(v_{n}\right)=0$. Using Ekeland's variational principle, there exists a $(P S)$ sequence $\left(u_{n}\right)$ for $I$ on $\mathcal{N}$ satisfying

$$
v_{n}(x)=u_{n}(x)+o_{n}(1) .
$$

By Lemmas 2.2 and 2.5, this sequence is bounded. If $m$ is not attained, Lemma $3.1 \mathrm{im}$ plies that there exists $\left(z_{n}\right) \subset \mathbb{R}^{N},\left|z_{n}\right| \rightarrow \infty$ and $u_{n}(x)=w\left(x-z_{n}\right)+o_{n}(1)$, where $w$ is the radial positive ground state solution of limit problem. Using the properties of the barycenter function, we obtain

$$
\beta\left(v_{n}\left(x+z_{n}\right)\right)=\beta\left(v_{n}\right)-z_{n}=-z_{n} \quad \text { and } \quad \beta\left(v_{n}\left(x+z_{n}\right)\right) \rightarrow \beta(w(x))=0,
$$

which is a contradiction, as we have $\left|z_{n}\right| \rightarrow \infty$.

Proof of Theorem 1.3. Initially, we assume that $m$ is attained for some $u \in \mathcal{N}$. Consequently, by Lemma 2.3, $u$ is a nontrivial solution of problem $(P)$. As such, consider that $m$ is not attained. So, by Lemma 5.1, we have that $m=m_{\infty}$ and there exists $\eta>0$ such that for all $u \in \mathcal{N}$ satisfying $I(u) \leq m_{\infty}+\eta$, then $\beta(u) \neq 0$. Fix $\eta$ in $\left(0, m_{\infty} / 8\right)$. By Lemma 4.6 , we have for all $R>R_{3}$

$$
I\left(T_{\lambda, y}^{R} z_{\lambda, y}^{R}\right) \leq m_{\infty}+\eta, \quad \forall y \in \partial B_{2}\left(y_{0}\right), \quad \lambda=0
$$

By Lemma 4.5, we can choose $\alpha$ in $\left(0, m_{\infty} / 8\right)$ and for all $R>R_{2}$

$$
I\left(T_{\lambda, y}^{R} z_{\lambda, y}^{R}\right) \leq 2 m_{\infty}-\alpha, \quad \forall y \in \partial B_{2}\left(y_{0}\right), \quad \lambda \in[0,1] .
$$

Consider $R>\max \left\{R_{2}, R_{3}\right\}$ and define the following map

$$
\begin{aligned}
& \mathcal{H}: \quad B_{2}\left(y_{0}\right) \rightarrow \mathcal{N} \cap I^{2 m_{\infty}-\eta}, \\
& \lambda y_{0}+(1-\lambda) y \rightarrow T_{\lambda, y}^{R} z_{\lambda, y}^{R} .
\end{aligned}
$$

The goal now is to show that $I$ has a critical value in $\left(m_{\infty}, 2 m_{\infty}\right)$. Arguing by contradiction, assume that is false. Since $m$ is not attained, by Lemma 3.2, I satisfies the PalaisSmale condition on $\mathcal{N}$ at any level in $\left(m_{\infty}, 2 m_{\infty}\right)$. Thus, there exists $\varepsilon>0$ such that

$$
\left\|I_{\mathcal{N}}^{\prime}(u)\right\| \geq \varepsilon \quad \text { for every } \quad u \in \mathcal{N} \cap I^{-1}\left[m_{\infty}+\eta, 2 m_{\infty}-\alpha\right] .
$$

This implies (see Lemma 5.15 [27]) there exists a continuous function (deformation)

$$
\mathcal{D}: \mathcal{N} \cap I^{2 m_{\infty}-\alpha} \longrightarrow \mathcal{N} \cap I^{m_{\infty}+\eta}
$$


such that $\mathcal{D}=i d$ for all $u \in \mathcal{N} \cap I^{m_{\infty}+\eta}$. Note that we can use a deformation on $\mathcal{N}$ which is a $C^{1}$ manifold due to Theorem 2.5 in [10], for instance. From (5.1), (5.2), (5.3) and (5.4), we can define

$$
\begin{aligned}
& \Gamma: B_{2}(0) \rightarrow \partial B_{2}(0), \\
& x \rightarrow A_{2}\left(2 \frac{\beta \circ \mathcal{D} \circ \mathcal{H} \circ A_{1}(x)}{\left|\beta \circ \mathcal{D} \circ \mathcal{H} \circ A_{1}(x)\right|}\right),
\end{aligned}
$$

where $\beta$ is the barycenter function and $A_{1}, A_{2}$ are the translations $A_{1}: B_{2}(0) \rightarrow B_{2}\left(y_{0}\right)$, $A_{1}(x)=x+y_{0}$ and $A_{2}: \partial B_{2}(0) \rightarrow \partial B_{2}(0), A_{2}(2 y /|y|)=y-y_{0}$, where $y \in \partial B_{2}\left(y_{0}\right) . \Gamma$ is a continuous function. In order to arrive at a contradiction, let us take $y \in \partial B_{2}\left(y_{0}\right)$, which means that $\lambda=0$. So, using the properties of the barycenter

$$
\beta\left(T_{0, y}^{R} z_{0, y}^{R}\right)=\beta\left(T_{0, y}^{R} w_{y}\right)=\beta\left(w_{y}\right)=\beta(w(x-R y))=\beta(w(x))+R y=R y .
$$

By (5.5) and (5.6), it holds

$$
\begin{aligned}
\Gamma\left(y-y_{0}\right) & =A_{2}\left(2 \frac{\beta \circ \mathcal{D} \circ \mathcal{H} \circ A_{1}\left(y-y_{0}\right)}{\left|\beta \circ \mathcal{D} \circ \mathcal{H} \circ A_{1}\left(y-y_{0}\right)\right|}\right) \\
& =A_{2}\left(2 \frac{\beta\left(T_{0, y}^{R} z_{0, y}^{R}\right)}{\left|\beta\left(T_{0, y}^{R} z_{0, y}^{R}\right)\right|}\right)=A_{2}\left(2 \frac{R y}{|R y|}\right)=y-y_{0} .
\end{aligned}
$$

Hence, we have a contradiction since such a continuous map $\Gamma$ does not exist by Brouwer's fixed point theorem.

Observe that $u$ does not change sign by Lemma 2.4. Since $f$ is an odd function, $-u$ is also solution of $\left(P_{a}\right)$. This gives the existence of a positive solution and the proof of the theorem is finished.

\section{Acknowledgements}

The first author is partially supported by CNPq/Brazil, PROEX/CAPES and FAPDF 0193.001765/2017. The research of the second author is partially supported by UFVJM.

The authors would like to thank Professor Yanyan Li for his suggestions of improvement in this article and his support.

\section{References}

[1] N. Ackermann, M. Clapp and F. Pacella, Alternating sign multibump solutions of nonlinear elliptic equations in expanding tubular domains, Commun. Partial Differential Equations, 38 (2013), 751-779.

[2] N. Akhmediev, W. Królinowski and A. Snyder, Partially coherent solitons of variable shape, Phys. Rev. Lett., 81(21) (1998), pp. 4632-4635. 
[3] A. Ambrosetti and A. Malchiodi, Nonlinear Analysis and Semilinear Elliptic problems, Cambridge University Press, 2007.

[4] A. Bahri and P. L. Lions, On the existence of a positive solution of semilinear elliptic equations in unbound domain, Ann. Inst. Henri Poincaré, 14 (1997), 365-413.

[5] A. Bahri and Y. Y. Li, On a minimax procedure for the existence of a positive solution for certain scalar field, Revista Mat. Iberoamericana, 6(1-2), (1990).

[6] P. Bartolo, V. Benci and D. Fortunato, Abstract critical point theorems and applications to some nonlinear problems with "strong" resonance at infinity, Nonlinear Anal. Theory Methods Appl., 7 (1983), 981-1012.

[7] V. Benci and G. Cerami, Positive solutions of some nonlinear elliptic problems in exterior domains, Arch. Ration. Mech. Anal., 99(4) (1987), 283-300.

[8] H. Berestycki, T. Gallouet and O. Kavian, Equations de champs scalaires euclidiens non lineaires das le plan, C. R. Acad. Sci. Paris Ser. I Math., 297 (1983), 305-310.

[9] H. Berestycki and P. L. Lions, Nonlinear scalar field equations I. Existence of a ground state, Arch. Rational Mech. Anal., 82 (1983), 313-345.

[10] A. Bonnet, A deformation lemma on a $e^{1}$ manifold, Manuscripta Math., 81(3-4) (1993), 339359.

[11] G. Cerami and D. Passaseo, Existence and multiplicity results for semilinear elliptic Dirichlet problems in exterior domains, Nonlinear Anal. Theory Methods Appl., 24 (1995), 1533-1547.

[12] M. Clapp and L. A. Maia, A positive bound state for an asymptotically linear or superlinear Schrodinger equations, J. Differential Equations, 260 (2016), 3173-3192.

[13] W. Y. Ding and W. M. Ni, On the existence of positive entire solutions of a semilinear elliptic equation, Arch. Rational Mech. Anal., 91(4) (1986), 283-308.

[14] G. Évéquoz and T. Weth, Entire solutions to nonlinear scalar field equations with indefinite linear part, Adv. Nonlinear Studies, 12 (2012), 281-314.

[15] B. Gidas, W. M. Ni and L. Niremberg, Symmetry of positive solutions of nonlinear elliptic equations, Math. Analysis and Applications, Part A. Advances in Math. Supplementary Studies, 7A (1981).

[16] M. K. Kwong, Uniquess of positive solutions of $\Delta u-u+u^{p}=0$ in $\mathbb{R}^{N}$, Arch. Rational Mech. Anal., 105 (1989), 243-266.

[17] R. Lehrer and L. A. Maia, Positive solutions of asymptotically linear equations via Pohozaev manifold, J. Functional Anal., 266 (2014), 213-246.

[18] P. L. Lions, The concentration-compactness principle in the calculus of variations, The locally compact case, Ann. I. H. poincaré AN, 1 (1984), 109-145 and 223-283.

[19] L. A. Maia and B. Pellacci, Positive solutions for asymptotically linear problems in exterior domains, Annali di Matematica Pura ed Applicata, 196 (2017), 1399-1430.

[20] L. A. Maia and R. Ruviaro, Positive and nodal solutions of nonlinear Schrondinger equations in a saturable medium, Adv. Nonlinear Study, 15 (2015), 191-219.

[21] K. McLeod, Uniqueness of positive radial solutions of $\Delta u+f(u)=0$ in $\mathbb{R}^{N}$ II, Trans. Amer. Math. Soc., 339 (1993), 495-505.

[22] P. H. Rabinowitz, On a class of nonlinear Schrödinger equations, Z. Angew. Math. Phys., 43(2) (1992), 270-291.

[23] J. Serrin and M. Tang, Uniquess of ground states for quasilinear elliptic equations, Indiana Univ. Math., 49 (2000), 897-923.

[24] W. A. Strauss, Existence of solitary waves in higher dimensions, Commun. Math. Phys., 55 (1977), 149-162.

[25] C. A. Stuart, Guidance properties of nonlinear planar waveguides, Arch. Rational Mech. 
Anal., 125(2) (1993), 145-200.

[26] A. Szulkin and T. Weth, Ground state solutions for some indefinite variational problems, J. Functional Anal., 257 (2009), 3802-3822.

[27] M. Willem, Minimax Theorems, Volume 24, Birkhauser, Boston, 1996. 